\title{
Comparing the Postoperative Outcomes of Single-Incision Laparoscopic Appendectomy and Three Port Appendectomy With Enhanced Recovery After Surgery Protocol for Acute Appendicitis: A Propensity Score Matching Analysis
}

Won Jong Kim ${ }^{1}$, Hyeong Yong Jin ${ }^{1}$, Hyojin Lee ${ }^{1}$, Jung Hoon Bae ${ }^{1}$, Wooree Koh², Ji Yeon Mun ${ }^{1}$, Hee Ju Kim¹, In Kyu Lee ${ }^{1}$, Yoon Suk Lee ${ }^{1}$, Chul Seung Lee ${ }^{1}$

${ }^{1}$ Department of Surgery, Seoul St. Mary's Hospital, College of Medicine, The Catholic University of Korea, Seoul; ${ }^{2}$ Department of Surgery, Hansol Hospital, Seoul, Korea

Purpose: The objective of this study was to compare the perioperative outcomes between single-incision laparoscopic appendectomy (SILA) and 3-port conventional laparoscopic appendectomy (CLA) in enhanced recovery after surgery (ERAS) protocol.

Methods: Of 101 laparoscopic appendectomy with ERAS protocol cases for appendicitis from March 2019 to April 2020, 54 patients underwent SILA with multimodal analgesic approach (group 1) while 47 patients received CLA with multimodal analgesic approach (group 2). SILA and CLA were compared with the single institution's ERAS protocol. To adjust for baseline differences and selection bias, operative outcomes and complications were compared after propensity score matching (PSM).

Results: After 1:1 PSM, well-matched 35 patients in each group were evaluated. Postoperative hospital stays for patients in group 1 ( $1.2 \pm 0.8$ vs. $1.6 \pm 0.8$ days, $\mathrm{P}=0.037)$ were significantly lesser than those for patients in group 2 . However, opioid consumption $(2.0 \mathrm{mg}$ vs. $1.4 \mathrm{mg}, \mathrm{P}=0.1)$ and the postoperative scores of visual analogue scale for pain at 6 hours $(2.4 \pm 1.9 \mathrm{vs}$. $2.8 \pm 1.4, \mathrm{P}=0.260)$ and 12 hours $(2.4 \pm 2.0$ vs. $2.9 \pm 1.5, \mathrm{P}=0.257)$ did not show significant difference between the 2 groups. Conclusion: SILA resulted in shortening the length of hospitalization without increase in complications or readmission rates compared to CLA with ERAS protocol.

Keywords: Enhanced recovery after surgery; Appendectomy; Laparoscopy; Pain management; Postoperative pain

Received: Aug 10, 2020 • Revised: Sep 10,2020 • Accepted: Sep 15, 2020 Correspondence to: Chul Seung Lee, M.D., Ph.D.

Division of Colorectal Surgery, Department of Surgery, Seoul St. Mary's Hospital, College of Medicine, The Catholic University of Korea, 222 Banpodaero, Seocho-gu, Seoul 06591, Korea

Tel: +82-2-2258-6095, Fax: +82-2-595-2282

E-mail: Icscolonoscopy@gmail.com

ORCID: https://orcid.org/0000-0002-4859-3015

(C) 2021 The Korean Society of Coloproctology

This is an open-access article distributed under the terms of the Creative Commons Attribution NonCommercial License (https://creativecommons.org/licenses/by-nc/4.0) which permits unrestricted noncommercial use, distribution, and reproduction in any medium, provided the original work is properly cited.

\section{INTRODUCTION}

The enhanced recovery after surgery (ERAS) program has been widely adopted in laparoscopic colorectal surgery, as it reduces the risk of perioperative complications $[1,2]$. The elements of an ERAS protocol are based on the reduction of surgical stress [3]. Several studies demonstrated laparoscopic appendectomy for nonperforated appendicitis to be safely performed as fast-track protocols for children [4-6] and adults [7]. Recently, randomized controlled trials of laparoscopic appendectomy in appendicitis were conducted $[8,9]$ and the ERAS program for laparoscopic appendectomy has advantages over the traditional approach. 
Many studies conducted in the elective surgery setting have proved the safety and feasibility of ERAS programs, as well as their economic benefits [5]. However, most patients with appendicitis are admitted via the emergency room and the implementation of ERAS protocols in an emergency setting remains challenging [10, 11]. The preoperative carbohydrate treatment and optimizations, such as exercise and elimination of smoking or alcohol use, cannot be incorporated into ERAS protocol for the patients with appendicitis.

Single-incision laparoscopic appendectomy (SILA) is presently the most frequently performed single-incision laparoscopic procedure [12-16]. Surgical stress in SILA is theoretically lower than that in colon resection surgery. It can be safely performed even by a surgical resident with an appropriate training protocol [17]. However, evidence regarding the clinical effect of SILA combined with ERAS is lacking. In the present study, we developed ERAS protocol for appendectomy including complicated appendicitis. The purpose of this study was to compare the perioperative outcomes between SILA and conventional laparoscopic appendectomy (CLA) in ERAS protocol.

\section{METHODS}

\section{Patients}

Medical records of patients who underwent laparoscopic appendectomy for acute appendicitis at The Catholic University of Korea, Seoul St. Mary's Hospital from March 2019 to March 2020 were reviewed. We adapted and modified previously published ERAS and outpatient protocols to patients with acute appendicitis $[10,18-20]$. The ERAS management was the same between the 2 groups with all patients who underwent laparoscopic appendec- tomy following the ERAS protocol of our institution [21] (Fig. 1). A total of 101 eligible patients were identified and the patients were divided into 2 groups, the SILA ( $\mathrm{n}=54,53.5 \%$; group 1$)$ and CLA ( $\mathrm{n}=47,46.5 \%$; group 2$)$ groups. The choice of surgical procedure (SILA or CLA) was determined by the surgeon's preference. All patients in both groups received preoperative treatment with cefotetan $(1 \mathrm{~g})$ intravenously. It was first administered in the emergency department, and thereafter postoperatively. All patients with appendiceal perforation received concurrent intravenous metronidazole ( $50 \mathrm{mg} / \mathrm{kg}$ to a maximum dose of $2 \mathrm{~g} /$ day). Postoperatively, antibiotics were administered only in cases of complicated appendicitis, which was defined as evidence of perforation, gangrenous change of appendix, or abscess on operative finding.

Patients were enrolled in the emergency department after the diagnosis of acute appendicitis was made by a colorectal staff-surgeon. Inclusion criteria were patients aged between 15 and 80 years. Exclusion criteria were pregnant women, patients taking oral anticoagulants or immunosuppressive agents, patients with generalized peritonitis, patients with appendiceal abscess $(>4 \mathrm{~cm}$ ) requiring drainage, patients with other concomitant pathology (gynecologic, urologic, or other gastrointestinal disease) requiring additional surgical procedures, and patients who required open conversion. To adjust for baseline differences and selection bias, operative outcomes and complications were compared after propensity score matching (PSM).

Our study was approved by the Institutional Review Board of The Catholic University of Korea (KC20RISI0688). The requirement for informed consent was waived because of the retrospective nature of the study.

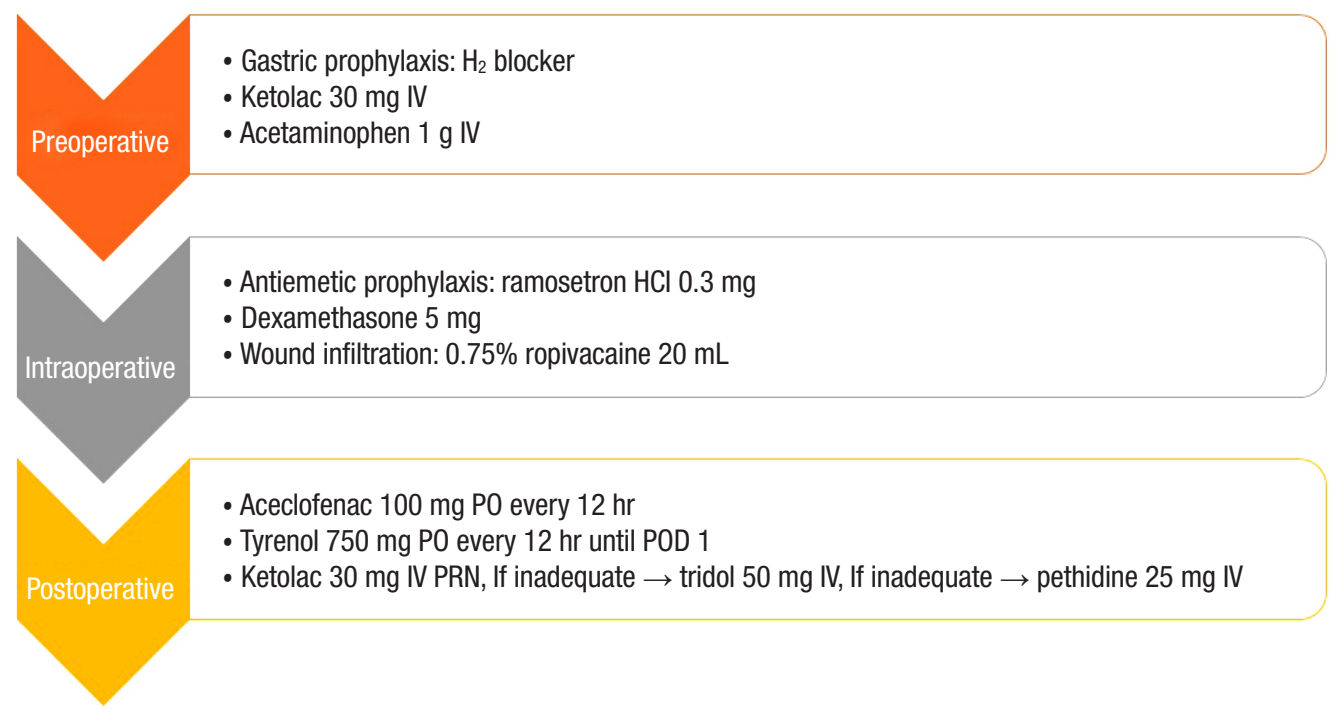

Fig. 1. Multimodal analgesia and injection for enhanced recovery after surgery protocol. IV, intravenous; HCI, hydrochloric acid; PO, per oral; POD, postoperative day; PRN, as needed. 


\section{Laparoscopic appendectomy with enhanced recovery after surgery protocol \\ Preoperative care}

The surgeon provided pain education to the patients including information concerning pain severity and type and a pain control plan. The patients were informed of the discharge criteria and the methods to control pain postoperatively, such as oral or intravenous medications as described previously [22]. Patients received preoperative treatment with intravenous opioid-sparing analgesia (acetaminophen $1 \mathrm{~g}$ and ketolac $30 \mathrm{mg}$ ).

\section{Intraoperative care}

Patients also received a single administration of ramosetron hydrochloric acid $0.3 \mathrm{mg}$ and dexamethasone $5 \mathrm{mg}$ at the end of the surgery. Just before the skin closure, 20 - $\mathrm{mL} 0.75 \%$ ropivacaine was infiltrated in the subcutaneous tissue at the umbilical wound site and $10 \mathrm{~mL}$ was injected at the left and right sides of the incision at the end of the surgery.

\section{Postoperative care}

Oral feeding with clear liquids was resumed when the patients were fully awakened after surgery. Opioid-sparing multimodal analgesia was administered (acetaminophen $750 \mathrm{mg}$ per oral [PO] and aceclofenac $100 \mathrm{mg}$ PO) (Fig. 1). Early ambulation was promoted. Patients were offered rescue analgesia with intravenous ketorolac $30 \mathrm{mg}$. If the effect of intravenous ketorolac was inadequate, intravenous opioid (tramadol $50 \mathrm{mg}$ or pethidine $25 \mathrm{mg}$ ) every 6 hours was administered as needed until patient discharge.

\section{Discharge}

Patients were discharged when a regular diet was tolerated, ambulation was achieved, and pain was adequately controlled with oral analgesics (visual analogue scale $[\mathrm{VAS}]<2$ ). At discharge, oral antibiotics (cefaclor) for a 3-day period were prescribed only to the patients with perforated appendicitis or peri appendiceal abscess. Opioid analgesics were not included in discharge medication.

\section{Operative technique}

All operations were performed by specialized colorectal surgeons with experience performing more than 100 SILA cases. SILA is performed according to the maneuver previously described [22]. Briefly, transumbilical access was created with open access. The commercial glove port (431AT-2W, Nelis, Bucheon, Korea) was then inserted into the peritoneal cavity. The appendectomy was performed using the standard laparoscopic 5-mm camera (full high definition laparoscope $5 \mathrm{~mm}$, Aesculap, Tullingen, Germany). A standard 3-port technique was employed with 1 of 12 -mm port and 2 of 5-mm ports. The appendix was ligated at the base with Vicryl Endoloops (Surgitie Ligating Loop, Covidien, Dublin, UK), and then divided. The transected appendix was extracted through the port after placing into the endoscopic retrieval bag (Lapbag, Sejong Medical Co., Paju, Korea). Appropriate irrigation with sa- line and hemostasis was ensured.

\section{Statistical analysis}

Statistical analysis was performed using IBM SPSS Statistics ver. 24.0 (IBM Corp., Armonk, NY, USA). Fisher exact test was used for categorical data and the Mann-Whitney U-test was used for continuous data. The results were presented as mean values and standard deviations for continuous normally distributed variables, as a median (interquartile range) for continuous nonnormally distributed data, and as counts and percentages for categorical data. To reduce the selection bias due to the retrospective design of the current study, PSM was conducted. To calculate the scores of the individuals, the nonparsimonious logistic regression model was used in accordance with the predefined covariates including sex, age, American Society of Anesthesiologists (ASA) physical status classification, body mass index (BMI), diagnosis (complicated appendicitis), or surgical procedure. The patients who underwent colorectal minimally invasive surgery were matched to others based on scores from algorithm of the nearest neighbor and 1:1 matching without specific caliper width or replacement. Multiple logistic regression was conducted to identify predictors for prolonged hospital stay. The analyzed independent variables were included due to a P-value of 0.05 or less on simple regression analysis with 1 independent variable. Subsequently, we performed stepwise backward variable elimination with threshold P-value of $>0.05$. P-values of $<0.05$ were considered statistically significant.

\section{RESULTS}

After 1:1 PSM, the well-matched 35 patients were evaluated in each group. As a result, differences in the demographics, including sex, age, ASA scores classification, BMI, and surgical procedure, were nonsignificant between the 2 groups (Table 1). Mean ages of patients in groups 1 and 2 were $40.2 \pm 13.6$ years and $36.0 \pm$ 19.2 years $(\mathrm{P}=0.295)$, respectively. Mean $\mathrm{BMI}$ in group 1 and 2 were $22.7 \pm 3.2 \mathrm{~kg} / \mathrm{m}^{2}$ and $23.1 \pm 3.9 \mathrm{~kg} / \mathrm{m}^{2}(\mathrm{P}=0.666)$, respectively. The median postoperative VAS scores at 6 hours (group 1, 2.4 \pm 1.9 ; group 2, $2.8 \pm 1.4 ; \mathrm{P}=0.260$ ) and 12 hours (group 1, 2.4 \pm 2.0 ; group $2,2.9 \pm 1.5 ; \mathrm{P}=0.257$ ) were not significantly different between the 2 groups (Table 2). Also, postoperative opioid consumption of group 1 was not significantly different from that of group 2 (group 1, $2.0 \pm 4.29 \mathrm{mg}$; group 2, $1.9 \pm 3.6 \mathrm{mg} ; \mathrm{P}=0.868$ ). However, the duration of postoperative hospital stays in the group 1 was significantly shorter than that of the group 2 (group 1, $1.2 \pm 0.8$; group 2, $1.6 \pm 0.8 ; \mathrm{P}=0.037$ ) (Table 2).

Postoperative complications were not statistically significantly different between the 2 groups. There were no open conversion, reoperations, or postoperative deaths in all groups (Table 2 ).

In the univariate analyses, long operation time (> 60 minutes), complicated appendicitis on initial computed tomography, high postoperative pain at 6 and 12 hours (VAS $>4$ ), and surgical type (CLA) were significantly associated with delayed postoperative 
Table 1. Patient demographics

\begin{tabular}{lccc}
\hline Variable & Group 1 $(\mathrm{n}=35)$ & Group 2 $(\mathrm{n}=35)$ & P-value \\
\hline Sex (male:female) & $18: 17$ & $22: 13$ & 0.334 \\
Age $(\mathrm{yr})$ & $40.2 \pm 13.6$ & $36.0 \pm 19.2$ & 0.295 \\
ASA PS classification & & & 0.356 \\
I & $30(85.7)$ & $27(77.1)$ & \\
$\|$ & $5(14.3)$ & $8(22.9)$ & \\
Body mass index $\left(\mathrm{kg} / \mathrm{m}^{2}\right)$ & $22.7 \pm 3.2$ & $23.1 \pm 3.9$ & 0.666 \\
Severity of appendicitis & & & 0.257 \\
$\quad$ Suppurative & $22(62.9)$ & $28(80.0)$ & \\
Perforated/gangrenous & $10(28.6)$ & $6(17.1)$ & \\
$\quad$ Abscess & \\
Preoperative WBC $(/ \mu \mathrm{L})$ & $3(8.6)$ & $1(2.9)$ & \\
Preoperative CRP $(\mathrm{mg} / \mathrm{L})$ & $3.2 \pm 5.5$ & $3.3 \pm 5.6$ & 0.931 \\
\hline
\end{tabular}

Values are presented as number only, mean \pm standard deviation, or number (\%). Group 1, single-incision laparoscopic appendectomy with multimodal analgesic approach; group 2, conventional laparoscopic appendectomy with multimodal analgesic approach. ASA, American Society of Anesthesiologists; PS, physical status; WBC, white blood cell; CRP, C-reactive protein.

aPeriappendiceal abscess.
Table 2. Perioperative outcomes

\begin{tabular}{lccc}
\hline Outcome & Group $1(\mathrm{n}=35)$ & Group 2 $(\mathrm{n}=35)$ & P-value \\
\hline Operative time (min) & $45.0 \pm 15.5$ & $53.0 \pm 32.8$ & 0.247 \\
Postoperative hospital stay (day) & $1.2 \pm 0.8$ & $1.6 \pm 0.8$ & 0.037 \\
Postoperative pain score (VAS) & & & \\
At $6 \mathrm{hr}$ & $2.4 \pm 1.9$ & $2.8 \pm 1.4$ & 0.260 \\
At $12 \mathrm{hr}$ & $2.4 \pm 2.0$ & $2.9 \pm 1.5$ & 0.257 \\
Total MMEs & $2.0 \pm 4.3$ & $1.9 \pm 3.6$ & 0.868 \\
Superficial/deep SSI (\%) & $1(2.9)$ & $0(0)$ & 0.314 \\
Organ space SSI (\%) & $0(0)$ & $1(2.9)$ & 0.314 \\
Ileus & $0(0)$ & $0(0)$ & $\mathrm{NA}$ \\
Severe complications & \\
Reoperation & $0(0)$ & $0(0)$ & $\mathrm{NA}$ \\
Readmission within 30 day & $0(0)$ & $0(0)$ & $\mathrm{NA}$ \\
Mortality within 30 day & $0(0)$ & $1(2.9)$ & 0.314 \\
\hline
\end{tabular}

Values are presented as mean \pm standard deviation or number $(\%)$.

Group 1, single-incision laparoscopic appendectomy with multimodal analgesic approach; group 2, conventional laparoscopic appendectomy with multimodal analgesic approach. VAS, visual analogue scale; MME, morphine milligram equivalents; SSI, surgical site infection. NA, not available.

${ }^{a}$ Clavien-Dindo classification $\geq$ Illa.

Table 3. Univariable and multivariable logistic regression analysis of predictors for prolonged hospital stay ( $>2$ days)

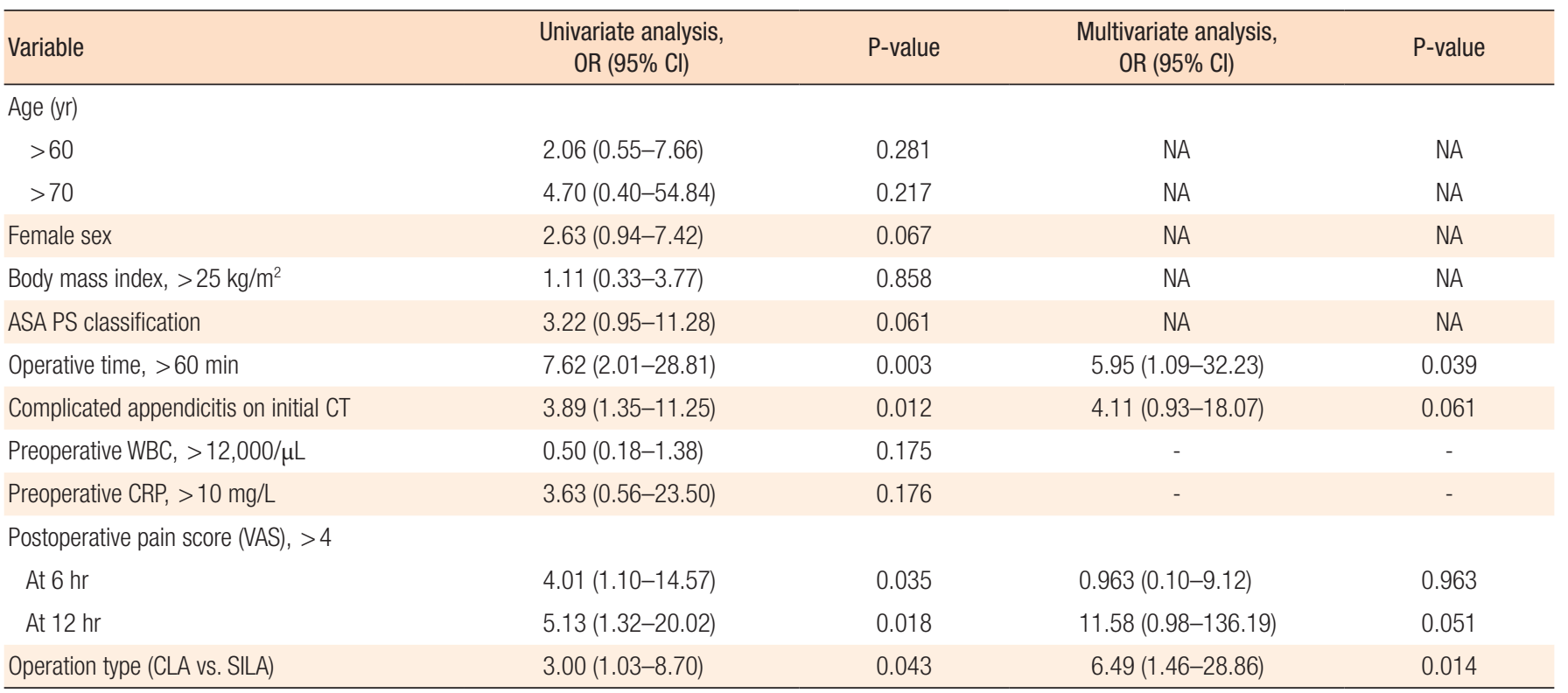

OR, odds ratio; Cl, confidence interval; NA, not available; ASA, American Society of Anesthesiologists; PS, physical status; CT, computed tomography; WBC, white blood cell; CRP, C-reactive protein; VAS, visual analogue scale; CLA, conventional laparoscopic appendectomy; SILA, single-incision laparoscopic appendectomy.

hospital length of stay. Moreover, in the multivariable analysis, long operation time ( $>60$ minutes) and surgical type (CLA) were independent risk factors for longer postoperative hospital stay $(\mathrm{P}=$ 0.039 and $\mathrm{P}=0.014$, respectively; Table 3 ).

\section{DISCUSSION}

Our findings demonstrated statistically significant differences in postoperative hospital stays, while other postoperative outcomes such as the operative time, 6- and 12-hours postoperative VAS, 
and postoperative complication rate were not different. Group 1 patients, who had received SILA with a multimodal pain protocol, had a reduced hospital stay compared to patients in group 2, who had received CLA with the multimodal pain protocol. Age of $>80$ years, immunosuppression, and the presence of serious comorbidities is associated with significantly worse prognosis for a benign disease, even in the absence of complications [23]. Therefore, we included the patients aged between 15 and 80 years.

In our study, the ERAS principles were applied to patients with both uncomplicated and complicated acute appendicitis undergoing laparoscopic appendectomy. The surgeries for patients with complicated appendicitis showing evidence of perforation and gangrenous appendixes in our study were more frequently performed in another worldwide study (perforation: 25 of 101, 24.8\% vs. 255 of 3,631,7.0\%) [24]. As our institution is a tertiary referral medical center, patients with simple appendicitis are often transferred to a local clinic whenever there is shortage of surgeons or anesthetists [22]. However, patients with severe appendicitis, such as perforated appendicitis, are likely to undergo surgery at our hospital.

Our ERAS program for patients included the preoperative pain education, effective pain control, opioid minimization, and early diet. Due to the diagnosis-related group (DRG) systems for patients undergoing appendectomy, some patients decline postoperatively to be discharged from the hospital until complete recovery; however, preoperative pain education has shortened the length of hospital stay after surgery [22].

Ropivacaine is an effective, long-acting local anesthetic administered perioperatively to reduce postoperative pain [25]. According to South Korea's DRG system, patients undergoing appendectomy cannot be charged for ultrasound [26]. Patients received ropivacaine in the subcutaneous tissue at the umbilical wound site at the end of the surgery eliminating the need for ultrasonography.

Poorly controlled postoperative pain could delay recovery, prolong a patient's hospital stay, and lower patient's satisfaction [23]. During postoperative recovery, reducing consumption of opioid is important to avoid opioid-related side effects and enhance recovery from surgery. Opioid-sparing analgesics have been successfully used in the perioperative setting, including nonsteroidal anti-inflammatory drugs (NSAIDs) and acetaminophen [27-29]. The postoperative pain was effectively controlled with opioid-sparing analgesics such as NSAIDs and acetaminophen. Narcotic analgesics were not used as pain relievers for discharge drugs.

In laparoscopic colon cancer treatment, a systemic approach, such as the ERAS program, appears to be more effective than a technical approach (single-port surgery) for significantly improving short-term surgical outcomes [30]. Similar to our finding, postoperative pain at 6 hours and 12 hours on the VAS scale was not significantly lower in group 1 than in group 2 within the ERAS program. Thus, ERAS protocol appears to be useful for improving the outcomes of the patients undergoing both SILA and CLA.

In contrast, the patients who received SILA with ERAS had reduced length of hospital stay. Despite, preoperative pain educa- tion and discharge criteria, some patients decline to be discharged postoperatively from the hospital until complete recovery, even when no longer symptomatic in terms of pain and fatigue. SILA may affect patient satisfaction, including cosmetic effects, and consequently, the number of patients who refuse to be discharged while meeting the discharge requirements can be reduced.

Recently, the World Society of Emergency Surgery recommended the use of broad-spectrum antibiotics in case of complicated acute appendicitis for a minimum duration of 3 to 5 days [23], and no postoperative antibiotics for uncomplicated appendicitis. In a largescale observational study, the most commonly used antibiotic was metronidazole (58.2\%) followed by second- and third-generation cephalosporins (37.0\% and $17.2 \%$, respectively) [23]. In this study, $39.6 \%$ (40 of 101) of the complicated acute appendicitis cases used 5 days of antibiotic treatment such as metronidazole and thirdgeneration cephalosporins while the uncomplicated appendicitis cases did not use postoperative antibiotics.

The reported incidence of postoperative complications in literature ranges from $3 \%$ to $28.7 \%$ [31, 32]. The common complications quoted in the literature are small bowel obstruction, surgical site infection, stump leakage, abdominal abscess, and stump appendicitis $[33,34]$. In this study, no severe complication of small bowel obstruction, stump leakage, or stump appendicitis were reported in both groups.

This study had some limitations. Since this is a retrospective study, selection bias cannot be avoided. The choice of surgical method for SILA or CLA during the study period was determined by the surgeon's preference. Patients who underwent laparoscopic appendectomy with residents had a higher incidence of serious and overall morbidity and longer surgical times than those who underwent laparoscopic appendectomy without residents [34]. To overcome this bias, all operations were performed by specialized colorectal surgeons. Also, an unprejudiced comparison was attempted using PSM analysis. Finally, our study did not compare the cost effectiveness between the 2 groups. According to the price approved by the Korean Health Insurance, a glove port and conventional 3 -port (1 of 12 -mm port and 2 of 5 -mm ports) cost 250 US dollars (USD) and 290 USD respectively. Therefore, in terms of medical expense SILA is not likely to be more expensive compared to CLA. However, this is an estimation based on only the port price, further trials including other medical expenses (i.e., dressing materials) should be done to compare the cost effectiveness of the 2 groups.

In conclusion, results from this study suggest that the ERAS protocol is feasible and useful for improving the outcomes of the patients undergoing SILA. It was a significant factor for reducing the length of hospital stay within the ERAS protocol. SILA with ERAS protocol has further benefits of shorter postoperative hospital stays and increased patient satisfaction. Further research, in the form of prospective, multicenter, randomized controlled trials, is needed to validate the advantage of SILA within the ERAS protocol. 


\section{CONFLICT OF INTEREST}

No potential conflict of interest relevant to this article was reported.

\section{REFERENCES}

1. Kehlet H. Multimodal approach to control postoperative pathophysiology and rehabilitation. Br J Anaesth 1997;78:606-17.

2. Gustafsson UO, Scott MJ, Hubner M, Nygren J, Demartines N, Francis N, et al. Guidelines for perioperative care in elective colorectal surgery: Enhanced Recovery After Surgery $\left(\right.$ ERAS $\left.^{\circledR}\right)$ Society Recommendations. 2018. World J Surg 2019;43:659-95.

3. Garulli G, Lucchi A, Berti P, Gabbianelli C, Siani LM. "Ultra" E.R. A.S. in laparoscopic colectomy for cancer: discharge after the first flatus? A prospective, randomized trial. Surg Endosc 2017;31:180613.

4. Grewal H, Sweat J, Vazquez WD. Laparoscopic appendectomy in children can be done as a fast-track or same-day surgery. JSLS 2004;8:151-4.

5. Trevino CM, Katchko KM, Verhaalen AL, Bruce ML, Webb TP. Cost effectiveness of a fast-track protocol for urgent laparoscopic cholecystectomies and appendectomies. World J Surg 2016;40:85662.

6. Aguayo P, Alemayehu H, Desai AA, Fraser JD, St Peter SD. Initial experience with same day discharge after laparoscopic appendectomy for nonperforated appendicitis. J Surg Res 2014;190:93-7.

7. Grigorian A, Kuza CM, Schubl SD, Nguyen NT, de Virgilio C, Kim $\mathrm{D}$, et al. Same-day discharge after non-perforated laparoscopic appendectomy is safe. J Invest Surg 2019 Jun 20 [Epub]. https:// doi.org/10.1080/08941939.2019.1630065.

8. Nechay T, Sazhin A, Titkova S, Tyagunov A, Anurov M, MelnikovMakarchuk K, et al. Evaluation of enhanced recovery after surgery program components implemented in laparoscopic appendectomy: prospective randomized clinical study. Sci Rep 2020;10:10749.

9. Trejo-Avila ME, Romero-Loera S, Cardenas-Lailson E, Blas-Franco M, Delano-Alonso R, Valenzuela-Salazar C, et al. Enhanced recovery after surgery protocol allows ambulatory laparoscopic appendectomy in uncomplicated acute appendicitis: a prospective, randomized trial. Surg Endosc 2019;33:429-36.

10. Cash CL, Frazee RC, Abernathy SW, Childs EW, Davis ML, Hendricks JC, et al. A prospective treatment protocol for outpatient laparoscopic appendectomy for acute appendicitis. J Am Coll Surg 2012;215:101-5.

11. Gustafsson UO, Scott MJ, Schwenk W, Demartines N, Roulin D, Francis N, et al. Guidelines for perioperative care in elective colonic surgery: Enhanced Recovery After Surgery $\left(\right.$ ERAS $\left.^{\circledR}\right)$ Society recommendations. Clin Nutr 2012;31:783-800.

12. Kim JH, Kim HY, Park SK, Lee JS, Heo DS, Park SW, et al. Singleincision laparoscopic appendectomy versus conventional laparoscopic appendectomy: experiences from 1208 cases of single-incision laparoscopic appendectomy. Ann Surg 2015;262:1054-8.

13. Duza G, Davrieux CF, Palermo M, Khiangte E, Azfar M, Rizvi
SAA, et al. Conventional laparoscopic appendectomy versus single-port laparoscopic appendectomy, a multicenter randomized control trial: a feasible and safe alternative to standard laparoscopy. J Laparoendosc Adv Surg Tech A 2019;29:1577-84.

14. Lee WS, Choi ST, Lee JN, Kim KK, Park YH, Lee WK, et al. Single-port laparoscopic appendectomy versus conventional laparoscopic appendectomy: a prospective randomized controlled study. Ann Surg 2013;257:214-8.

15. Frutos MD, Abrisqueta J, Lujan J, Abellan I, Parrilla P. Randomized prospective study to compare laparoscopic appendectomy versus umbilical single-incision appendectomy. Ann Surg 2013; 257:413-8.

16. Perez EA, Piper H, Burkhalter LS, Fischer AC. Single-incision laparoscopic surgery in children: a randomized control trial of acute appendicitis. Surg Endosc 2013;27:1367-71.

17. Lee GR, Kim JH, Kim CH, Lee YS, Kim JJ. Single-incision laparoscopic appendectomy is a safe procedure for beginners to perform: experience from 1948 cases. Surg Endosc 2020 Jun 16 [Epub]. https://doi.org/10.1007/s00464-020-07744-9.

18. Gustafsson UO, Scott MJ, Schwenk W, Demartines N, Roulin D, Francis N, et al. Guidelines for perioperative care in elective colonic surgery: Enhanced Recovery After Surgery $\left(\operatorname{ERAS}\left({ }^{\circledR}\right)\right)$ Society recommendations. World J Surg 2013;37:259-84.

19. Dubois L, Vogt KN, Davies W, Schlachta CM. Impact of an outpatient appendectomy protocol on clinical outcomes and cost: a case-control study. J Am Coll Surg 2010;211:731-7.

20. Lasso Betancor CE, Ruiz Hierro C, Vargas Cruz V, Orti Rodriguez RJ, Vazquez Rueda F, Paredes Esteban RM. Implementation of "fast-track" treatment in paediatric complicated appendicitis. Cir Pediatr 2013;26:63-8.

21. Kim WJ, Mun JY, Kim HJ, Yoon SH, Han SR, Bae JH, et al. Surgical rectus sheath block combined with multimodal pain management reduces postoperative pain and analgesic requirement after single-incision laparoscopic appendectomy: a retrospective study. Int J Colorectal Dis 2021;36:75-82.

22. Seo JW, Kim MJ, Yoon SH, Paik KY, Park SM, Kang WK, et al. The effects of preoperative pain education on the decision to discharge patients following single-incision laparoscopic appendectomy. Ann Coloproctol 2020 Jan 24 [Epub]. https://doi.org/10.3393/ac.2020. 01.16 .

23. Di Saverio S, Birindelli A, Kelly MD, Catena F, Weber DG, Sartelli $\mathrm{M}$, et al. WSES Jerusalem guidelines for diagnosis and treatment of acute appendicitis. World J Emerg Surg 2016;11:34.

24. Sartelli M, Baiocchi GL, Di Saverio S, Ferrara F, Labricciosa FM, Ansaloni L, et al. Prospective Observational Study on acute Appendicitis Worldwide (POSAW). World J Emerg Surg 2018;13:19.

25. Hansen TG. Ropivacaine: a pharmacological review. Expert Rev Neurother 2004;4:781-91.

26. Kim KH, Lee SC, Lee SK, Choi BJ, Jeong W, Kim SJ. Does Korea’s current diagnosis-related group-based reimbursement system appropriately classify appendectomy patients? Ann Surg Treat Res 2016;91:66-73. 
27. Wick EC, Grant MC, Wu CL. Postoperative multimodal analgesia pain management with nonopioid analgesics and techniques: a review. JAMA Surg 2017;152:691-7.

28. Dahl JB, Nielsen RV, Wetterslev J, Nikolajsen L, Hamunen K, Kontinen VK, et al. Post-operative analgesic effects of paracetamol, NSAIDs, glucocorticoids, gabapentinoids and their combinations: a topical review. Acta Anaesthesiol Scand 2014;58:1165-81.

29. Tan M, Law LS, Gan TJ. Optimizing pain management to facilitate Enhanced Recovery After Surgery pathways. Can J Anaesth 2015;62:203-18.

30. Kim MK, Kim JG, Lee G, Won DD, Lee YS, Kye BH, et al. Comparison of the effects of an ERAS program and a single-port laparoscopic surgery on postoperative outcomes of colon cancer patients. Sci Rep 2019;9:11998.

31. Emil S, Elkady S, Shbat L, Youssef F, Baird R, Laberge JM, et al. Determinants of postoperative abscess occurrence and percuta- neous drainage in children with perforated appendicitis. Pediatr Surg Int 2014;30:1265-71.

32. Isaksson K, Montgomery A, Moberg AC, Andersson R, Tingstedt B. Long-term follow-up for adhesive small bowel obstruction after open versus laparoscopic surgery for suspected appendicitis. Ann Surg 2014;259:1173-7.

33. Gandaglia G, Ghani KR, Sood A, Meyers JR, Sammon JD, Schmid $\mathrm{M}$, et al. Effect of minimally invasive surgery on the risk for surgical site infections: results from the National Surgical Quality Improvement Program (NSQIP) Database. JAMA Surg 2014;149: 1039-44.

34. Advani V, Ahad S, Gonczy C, Markwell S, Hassan I. Does resident involvement effect surgical times and complication rates during laparoscopic appendectomy for uncomplicated appendicitis? An analysis of 16,849 cases from the ACS-NSQIP. Am J Surg 2012; 203:347-51. 